\title{
Individualized 3D printed model-assisted posterior screw fixation for the treatment of craniovertebral junction abnormality: a retrospective study
}

\author{
Fangyou Gao, MD, Qu Wang, MD, Chuangxi Liu, MS, Bing Xiong, MS, and Tao Luo, MS \\ Department of Neurosurgery, Guizhou Provincial People's Hospital, Guizhou Province, China
}

OBJECTIVE This study was undertaken to evaluate the feasibility and efficacy of individualized 3D printed model-assisted posterior internal fixation in treating craniovertebral junction (CVJ) abnormalities.

METHODS Forty-four patients (19 males and 25 females; mean age $36.5 \pm 9.2$ years, range 11-62 years; symptom duration 1 month-15 years) with a CVJ abnormality who were admitted to the authors' institution between April 2010 and April 2015 were retrospectively studied. The individualized 3D printed model of the CVJ was produced based on the individual CT data by use of 3D printing technology. The posterior internal fixation was simulated on the model to obtain data for individual patients, which were then used for intraoperative assistance. One-stage posterior decompression combined with internal fixation was performed. The results were evaluated using the Japanese Orthopaedic Association (JOA) scale, atlanto-dens interval (ADI), and cervicomedullary angle (CMA). The patients underwent follow-up and outcomes were evaluated using $\mathrm{CT}, \mathrm{MRI}$, and the JOA scale score. The comparison between preoperative and postoperative JOA scale scores was done using a paired t-test.

RESULTS Thirty-eight individualized 3D printed models were successfully built. The 38 patients underwent successful posterior internal fixation performed with the assistance of 3D printed models. In the 6 patients without an individualized printed model (i.e., the patients who underwent surgery before 3D printed modeling was available at the authors' institution), the pedicle screw was inserted incorrectly into the transverse foramen in 2 patients and inserted incorrectly into the vertebral canal in 1 patient. All patients were observed for a mean of 26 months (range 3-52 months). The postoperative JOA scale scores for all patients significantly improved from the preoperative scores. Among the 41 patients treated with atlantoaxial distraction reduction, postoperative MRI and CT showed complete reduction in 31 patients and incomplete reduction in 10 patients (reduction rate $>50 \%$ ). The postoperative ADI significantly decreased, and the CMA significantly increased.

CONCLUSIONS Individualized 3D printed model-assisted posterior internal fixation seems feasible and effective in optimizing the treatment of $\mathrm{CVJ}$ abnormalities. In addition, it offers many advantages, including preoperative simulation, intraoperatve guidance, and intraoperative error minimization.

https://thejns.org/doi/abs/10.3171/2016.11.SPINE16713

KEY WORDS 3D printed model; atlantoaxial dislocation; craniovertebral junction abnormality; internal fixation; surgical technique

$\mathrm{C}$ RANIOVERTEBRAL junction (CVJ) abnormality is a congenital or acquired condition characterized by a variety of osseous and neurological deformities.9.13 Surgery is the main treatment method. Patients without CVJ instability can be treated with posterior decompression. Patients with CVJ instability are usually treated with internal fixation, especially those with atlantoaxial dislocation (AAD). ${ }^{6}$ Generally, posterior internal fixation with $\mathrm{C}-2$ pedicle screws is the most commonly used method. In addition to the different individual bone structures, mul- tiple malformations often accompany CVJ abnormality, including variability of the vertebral artery (VA), which may increase operative difficulty and risks. Thus, the surgery is complex, especially for inexperienced surgeons. ${ }^{13}$ Individualized treatment is critical to achieving a satisfactory result. So far, no safe and reliable method exists that provides precise guidance for posterior internal fixation.

Surgeons usually depend on preoperative CT 3D imaging results and intraoperative judgment for screw implantation planning. Three-dimensional imaging is an im-

ABBREVIATIONS $\mathrm{AAD}=$ atlantoaxial dislocation; $\mathrm{ADI}=$ atlanto-dens interval; $\mathrm{AOA}=$ atlantooccipital assimilation; $\mathrm{CMA}=\mathrm{cervicomedullary}$ angle $; \mathrm{CVJ}=\mathrm{craniovertebral}$ junction; JOA = Japanese Orthopaedic Association; VA = vertebral artery.

SUBMITTED April 19, 2016. ACCEPTED November 21, 2016.

INCLUDE WHEN CITING Published online May 5, 2017; DOI: 10.3171/2016.11.SPINE16713. 
portant clinical tool for planning complex surgeries and is considered to be superior to conventional $2 \mathrm{D}$ imaging when visualizing anatomical structures, ${ }^{8}$ given that the 3D image shows the vertebral body in different views. However, the $3 \mathrm{D}$ images are viewed on a $2 \mathrm{D}$ computer screen, which is a disadvantage in guiding the surgery. The anatomical structure is extremely complex with many individual variations, especially among patients with CVJ deformity at C-2.

Three-dimensional printing systems convert individual CT data into life-sized 3D models, mimicking real tissue and surgical procedures.,10 These individualized 3D models can assist in intraoperative navigation. With individualized 3D models, surgeons are able to identify the anatomical structures, which may optimize preoperative surgical planning, thus allowing surgeons to avoid intraoperative mistakes and reduce possible surgical complications, including VA injury.

Individualized 3D models have been used in the field of kidney transplantation and pediatric otolaryngology, ${ }^{4,11}$ but their use has rarely been reported in the field of CVJ abnormality. In this study, we evaluated the feasibility and efficacy of the use of individualized 3D printed models to facilitate the surgical treatment of CVJ abnormalities. The Japanese Orthopaedic Association (JOA) scale score, atlanto-dens interval (ADI), and cervicomedullary angle (CMA) were used to evaluate the results.

\section{Methods \\ Patients}

This study was approved by the ethics committee of Guizhou Provincial People's Hospital, and signed informed consent was obtained from all patients. Forty-four patients (19 males and 25 females; mean age $36.5 \pm 9.2$ years, range 11-62 years; symptom duration 1 month-15 years) with CVJ abnormalities who were admitted to the hospital between April 2010 and April 2015 were enrolled in the study. Clinical manifestations included craniovertebral pain ( 29 cases), incomplete paralysis (34 cases), hemiplegia (4 cases), gait disturbance (18 cases), numbness and weakness in bilateral upper limbs (11 cases), numbness and weakness in unilateral upper limb (5 cases), expiratory dyspnea (4 cases), trachyphonia with dysphagia (2 cases), torticollis (4 cases), diplopia (1 case), and thenar muscle atrophy (6 cases). All patients underwent radiography, MRI, and CT scanning of the CVJ. The patients were diagnosed as having a CVJ abnormality, which included atlantoaxial dislocation (AAD) in 41 cases, Arnold-Chiari malformation in 20 cases, atlantooccipital assimilation (AOA) in 39 cases, basilar invagination in 33 cases, syringomyelia in 12 cases, platybasia in 5 cases, and an incomplete cervical segment in 10 cases. Each patient presented with 2-6 of the aforementioned abnormalities. The inclusion criteria were instability in the craniocervical junction region according to radiographic, CT, and MRI examination results.

\section{Individualized 3D Model and Simulation of the Surgery}

Preoperatively, thin-slice CT scanning (range 0.6-0.75 $\mathrm{mm}$ ), image reconstruction, and data collection were performed. Forty-one patients underwent CT angiography for VA reconstruction. DICOM data were entered into the Aquarius iNtuition Viewer software (TeraRecon, Inc.) to construct a 3D image of the CVJ. The preliminary preoperative evaluation was performed. Electronic simulation of screw implantation was done using Mimics Innovation Suite (Materialise). Thirty-eight individualized 3D printed models were successfully built. Based on the individualized 3D printed model, further evaluation of the abnormality and treatment method was done, with a focus on the atlantoaxial portion. Simulation of screw implantation was also performed on the 3D printed model (see Fig. 3L). Individualized data for screw implantation were obtained to guide the internal fixation treatment.

\section{Operation}

If the patient presented with AAD, surgery was performed as described by Jian et al., ${ }^{3}$ with some modifications. Posterior decompression was performed. Occiput$\mathrm{C} 2$ pedicle screw distraction reduction and internal fixation were performed. In patients without dislocation, internal fixation in situ and bone graft fusion were performed after the posterior decompression.

The posterior internal fixation procedure was performed according to preoperative radiography, CT, and MRI findings; the individualized 3D printed model; and intraoperative judgment. In patients who did not present with AOA, internal fixation was performed using a C-1 lateral mass screw, a C-2 pedicle screw (ZhengTian Medical Instruments Co.), and a C1-2 titanium rod (diameter, 3.0 $\mathrm{mm}$; DePuy Spine or ZhengTian Medical Instruments Co.) if the bone condition of C-1 and C-2 permitted. In patients who presented with AOA, internal fixation was performed using occipital and C-2 pedicle screws and titanium rods. If the patient presented with C-2 pedicle dysplasia or if pedicle screw implantation was unsuitable, lamina nailing or isthmus nailing was performed; C-3 lateral mass screw fixation (occiput-C2-C3) was added if necessary.

General anesthesia was induced, and the patient was placed prone with the head fixed in the head holder. An incision was made in the posterior center of the occipital cervix. The screw insertion site and the exposure range were determined according to the 3D printed model. The VA was carefully protected. Decompression was performed at the posterior border of the foramen magnum. The scope of the bone removal was determined according to the imaging results and the 3D printed model. Screw placement, installation of the Y-shaped occipital plate (width, $31 \mathrm{~mm}$; ZhengTian Medical Instruments Co.), and rod fixation (diameter, $3.0 \mathrm{~mm}$; DePuy Spine or ZhengTian Medical Instruments Co.) were performed. If AAD was present, craniovertebral fixation was done. The $\mathrm{C}-2$ pedicle screw was fastened, and distraction reduction was performed. After complete reduction, all nuts were fastened. The bone graft was implanted, and the incision was closed.

\section{Evaluation of Results and Follow-Up}

Outcomes of posterior internal fixation were evaluated using the JOA scale. As for the patients with AAD who were treated with distraction reduction, the effect of the distraction reduction was evaluated according to ADI and 

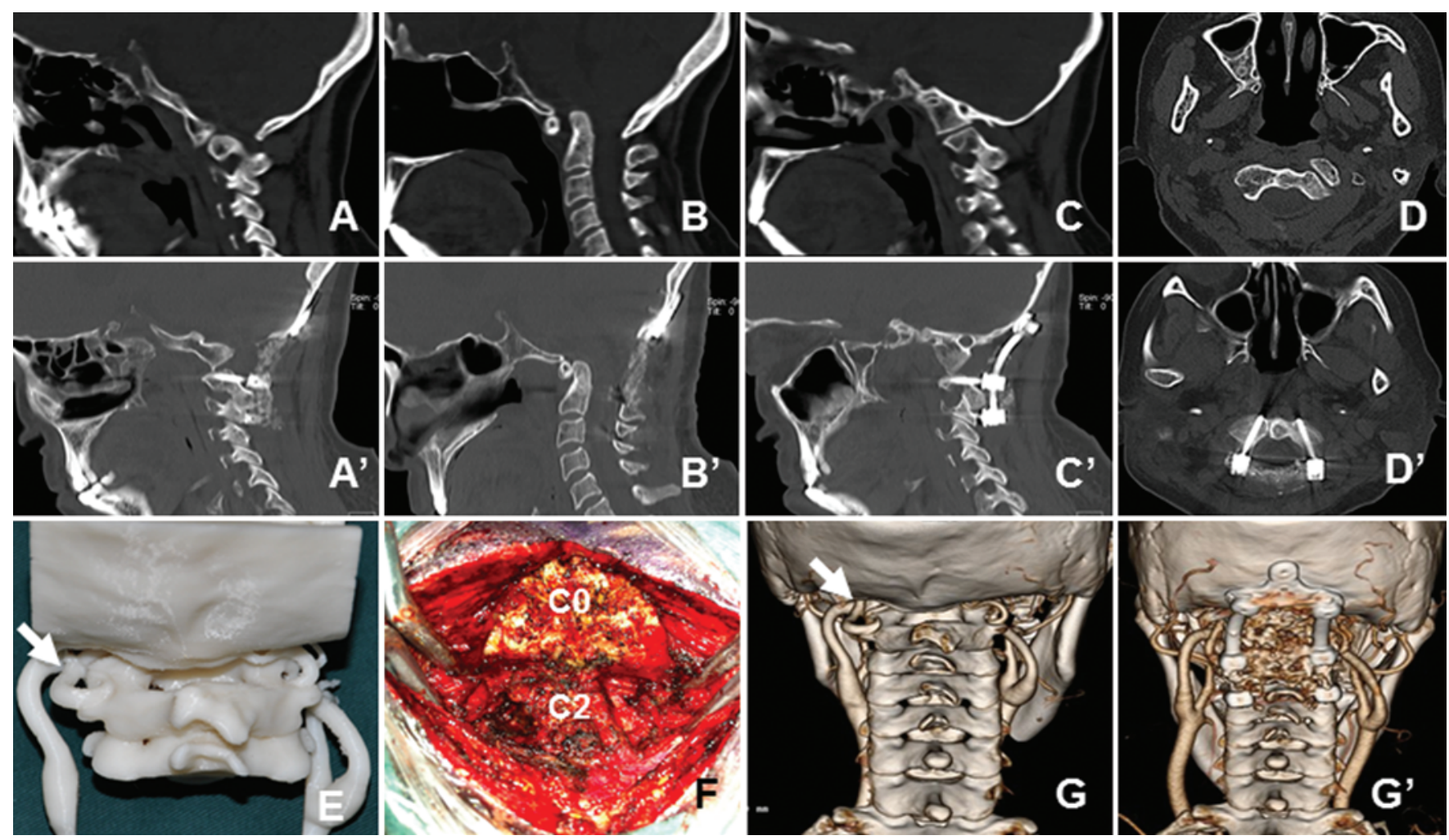

FIG. 1. Individualized 3D printed model-assisted posterior screw fixation in a 55-year-old woman with AAD. A-D: Reconstructed $3 D$ sagittal $(A-C)$ and axial (D) CT images showing $A A D$ and a thin bilateral $C-2$ pedicle, indicating that screw implantation is impossible. A'-D': Postoperative reconstructed CT images. According to the 3D printed model, screw implantation in the pedicle combined with $C$-3 lateral mass screw placement could be performed. Sagittal $\left(A^{\prime}-C^{\prime}\right)$ and axial $\left(D^{\prime}\right)$ images showing the normal neck after the screw implantation and the reduction. E: The individualized 3D printed model including the VAs. The arrow in panels $\mathrm{E}$ and $\mathrm{G}$ indicates the abnormal left VA. F: Intraoperative photograph showing the occipital bone (C0) and the $\mathrm{C}-2$ spinous process and lamina, as is consistent with the model. $\mathbf{G}$ and $\mathbf{G}^{\prime}$ : Preoperative $(G)$ and postoperative $\left(G^{\prime}\right) 3 D$ CT images show that the bilateral VAs are normal without any injury. Figure is available in color online only.

CMA based on results of the CT 3D reconstructed image and the sagittal T2-weighted MR image.

All patients underwent follow-up. One week postoperatively, CT and MRI examination were performed, and the ADI and CMA measurements were recorded. Three months after the surgery, the JOA scale score was recorded to assess the therapeutic results. The complications were also recorded.

\section{Statistical Analysis}

Data are expressed as the mean \pm SD. The paired t-test was performed to compare the preoperative and postoperative data using SPSS software (version 19.0, IBM); $\mathrm{p}<$ 0.05 was considered statistically significant.

\section{Results}

CVJ 3D CT Reconstruction and Printed Models With the VA

Among the 41 cases for which 3D VA reconstruction was obtained, there were 9 cases with a high-riding VA, 7 cases with an abnormal artery course and without VA loops, and 5 cases with a thin or unilaterally absent VA and contralateral compensatory enlargement. In 38 of these cases, individualized 3D printed models were obtained (Figs. 1E, 2G, and 3L).

\section{Surgical Results and Complications}

Three patients without AAD underwent direct posterior internal fixation. The remaining 41 patients with AAD underwent posterior distraction reduction (Figs. 2 and 3A$\mathrm{H})$. Postoperatively, torticollis and cervical scoliosis were mostly corrected (Fig. 3I-K). There were 34 cases of occiput-C2 fixation, 6 cases of occiput-C2-C3 fixation, and 4 cases of $\mathrm{C} 1-2$ fixation. In the 38 patients for whom 3D printed models were made, the surgery was successfully performed without any accidents or intraoperative mistakes such as VA injury (Fig. $1 \mathrm{G}$ and $\mathrm{G}^{\prime}$ ). The remaining 6 patients did not have a 3D printed model because they underwent surgery before 3D printing was available at our institution. Two of these 6 patients without an individualized printed model had a pedicle screw that had been incorrectly inserted into transverse foramen during unilateral C-2 pedicle screw placement. Of these 2 patients, one was treated with reducing the screw insertion depth without serious postoperative complications, and the other was treated with lamina screw fixation. The pedicle screw was inserted incorrectly into the vertebral canal in 1 case, and the nerve root was injured, resulting in postoperative numbness in the unilateral upper limb.

As for the surgery planning, according to the preoperative CT results it seemed possible that 2 patients could 

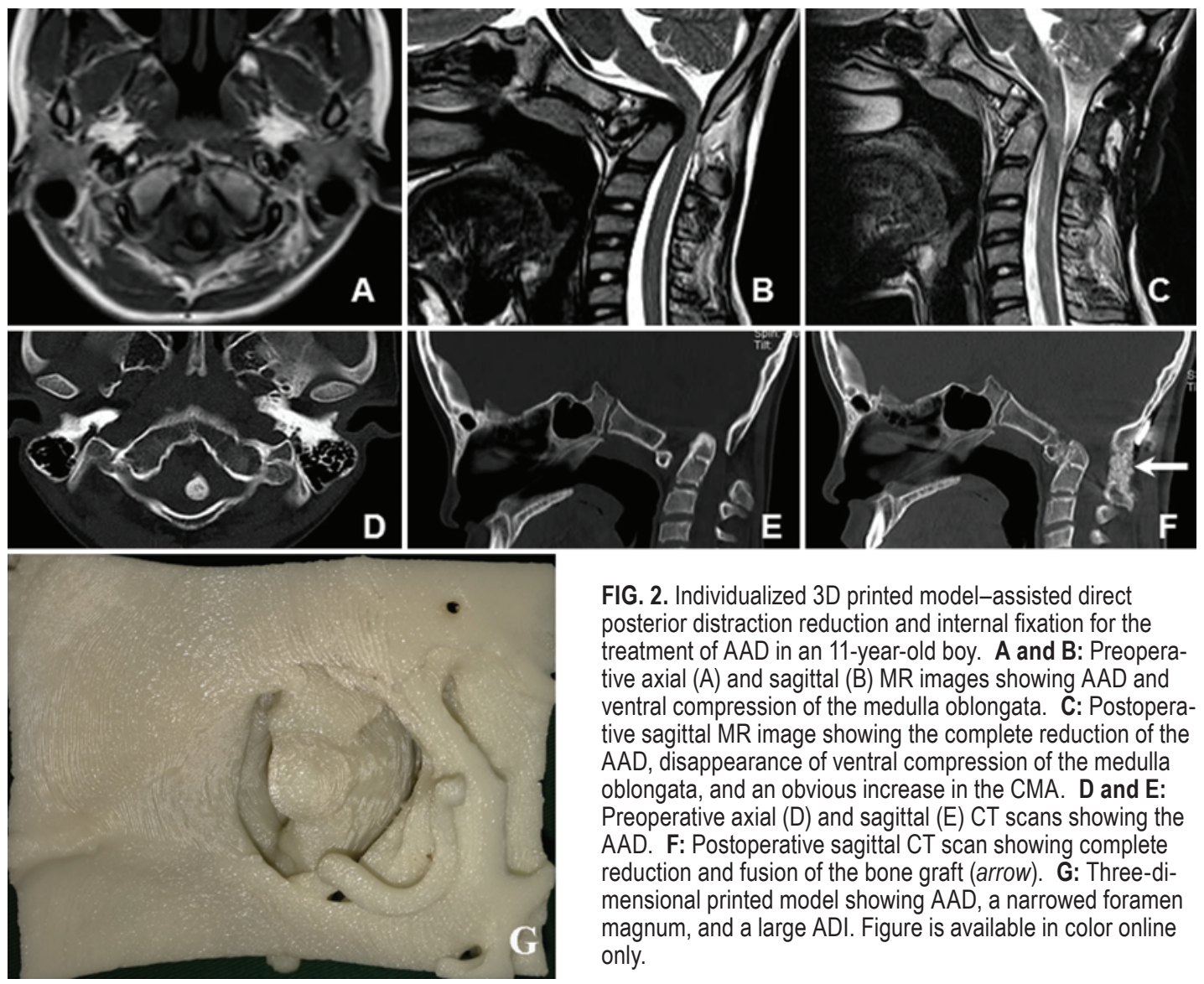

FIG. 2. Individualized 3D printed model-assisted direct posterior distraction reduction and internal fixation for the treatment of $A A D$ in an 11-year-old boy. A and B: Preoperative axial $(A)$ and sagittal $(B)$ MR images showing $A A D$ and ventral compression of the medulla oblongata. C: Postoperative sagittal MR image showing the complete reduction of the $A A D$, disappearance of ventral compression of the medulla oblongata, and an obvious increase in the CMA. D and E: Preoperative axial (D) and sagittal (E) CT scans showing the AAD. F: Postoperative sagittal CT scan showing complete reduction and fusion of the bone graft (arrow). G: Three-dimensional printed model showing $A A D$, a narrowed foramen magnum, and a large ADI. Figure is available in color online only.

have undergone treatment directly with $\mathrm{C}-2$ pedicle screw placement. However, the data from the 3D printed model showed that the pedicle was too thin for a long screw fixation. Therefore, short screws were inserted and C-3 lateral mass screw fixation was performed. In 3 cases, the preoperative 3D CT assessment suggested that the pedicle screw was not suitable due to a high-riding VA and should be performed using a lamina screw. However, the pedicle screw implantation was successfully performed after 3D printed model analysis and adjustment of the screw insertion site and direction (Fig. 1).

Two patients with AAD who underwent distraction reduction experienced inspiratory dyspnea $0.5-1$ hour after recovery from anesthesia and removal of the trachea cannula. These patients underwent immediate tracheotomy placement, and their breathing recovered to normal later. There were no operative deaths, VA injury, infection of the incision, screw loosing, or screw displacement.

\section{Follow-Up}

All patients underwent follow-up for a mean of 26 months (range 3-52 months). The postoperative JOA scale score significantly improved (Table 1). Among the 41 patients with AAD reduction, postoperative MRI and CT scanning showed complete reduction in 31 patients and incomplete reduction in 10 patients, with a reduction rate greater than $50 \%$. The postoperative ADI significantly decreased, and the CMA significantly increased (Table 1).
Ventral compression in the medulla oblongata was significantly reduced or disappeared.

\section{Discussion}

The individualized 3D printed model can be used to rehearse surgical procedures and thus provides additional information for presurgical plans and potentially reduces operative complications. ${ }^{12}$ The individualized model also ensures that the surgeons have a similar understanding of the anatomy in each case, thus increasing the likelihood of a successful surgery. As far as we know, this is the first report of the application of individualized 3D printed models to surgery for CVJ abnormalities. In the present study, surgery that was performed based on the individualized 3D printed models was successful, without any medical errors or complications. Preoperative simulation of screw placement on the individualized 3D printed model provides important guidance for the surgery and offers many advantages, because it is simple to make, convenient, and can be touched. Moreover, psychological pressure on the surgeons is reduced, especially for inexperienced surgeons.

Internal fixation treatment for AAD includes the methods of ligament release through the odontoid and fixation through the oral cavity (or via posterior fixation) ${ }^{14,15}$ and posterior atlantoaxial reduction combined with internal fixation. ${ }^{1}$ These methods are relatively complex and require intraoperative traction, which usually results in 

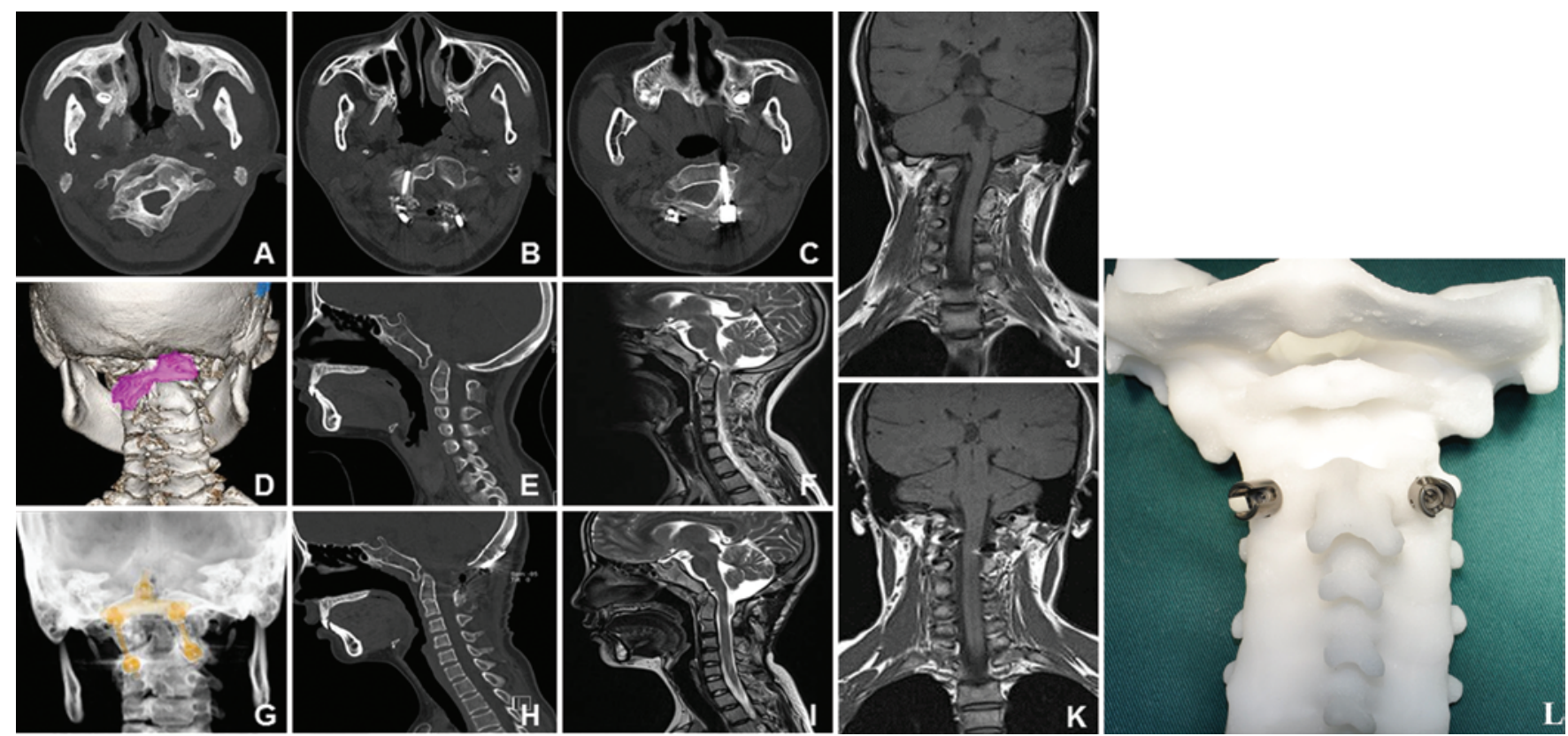

FIG. 3. Preoperative and postoperative CT and MR images obtained in an 18-year-old female patient with atlantoaxial rotation dislocation. The patient was treated with 3D printed model-assisted reduction. A-C: Axial reconstructed 3D CT images showing C-2 rotation dislocation and screw implantation. D: Reconstructed CT scan showing the seesaw appearance of C-2 rotation dislocation. E: Preoperative sagittal CT scan showing the increased ADI. F: Preoperative sagittal MR image showing ventral compression of the cervical cord, decreased CMA, and abnormality in cervical lateral bending. G: Coronal CT image of the postoperative internal fixation system. H: Postoperative sagittal CT image showing complete reduction of AAD. I: Postoperative sagittal MR image showing that the compression of cervical cord disappeared and torticollis and cervical scoliosis were corrected. $\mathrm{J}$ and $\mathrm{K}$ : Preoperative $(\mathrm{J})$ and postoperative $(\mathrm{K})$ coronal MR images showing that the torticollis and cervical scoliosis were corrected after the surgery. L: Preoperative simulation of screw implantation in the 3D model. Figure is available in color online only.

some complications. Jian et al. made significant improvements based on the traditional treatment mode. ${ }^{3}$ By use of posterior distraction reduction and posterior internal fixation, good therapeutic effect was obtained. In the present study some adjustments, such as to screw insertion sites and trajectories, were made based on analysis of the 3D printed model. For example, in the patient with C-2 rotation dislocation, the screw implantation site and direction were very different from those of the patient without rotation dislocation. Moreover, for the cases with rotation dislocation, torticollis and cervical scoliosis were corrected by adjusting the height of distraction reduction (Fig. 3).

When performing posterior internal fixation, the possibility of VA injury should be considered, as mentioned by Jian et al. ${ }^{3}$ Protection of the VA is critical for a successful surgery. The VA can follow a variable course, especially in patients with CVJ malformation. ${ }^{9}$ The variability may increase operative difficulty and risks. Empirical screw implantation is unreliable, with a high likelihood of VA injury. In the present study, there were 9 cases of a highriding VA, in which screw placement was difficult and the chance of VA injury was great. Five patients had an abnormal VA course that went beyond the operative field. It was easy to perform screw implantation in these patients with little possibility of injuring the VA. Five patients had a thinner VA laterally, and the operation was easy to perform on this side. However, due to the compensatory enlargement of the contralateral VA, careful consideration is of utmost importance when the operation is performed on the contralateral side. Individualized 3D printed models containing the VA can show the VA course and any abnormalities. Thus, use of the model for preplanning to avoid VA injury when placing the screw decreased the psychological burden on the surgeon. Through comparing the intraoperative anatomical structures with the $3 \mathrm{D}$ printed models, the surgeon was able to achieve the target while avoiding excessive exposure and reducing surgical difficulties and the possibility of VA injury. Injury to the VA did not occur in any patient with an individualized 3D printed model. In the 6 patients without an individualized 3D printed model, the screw was incorrectly inserted into the transverse foramen in 2 patients, indicating a high likelihood of VA injury.

Dyspnea is one of the serious complications that may occur after posterior reduction and internal fixation surgery for AAD. ${ }^{5}$ One study reported that a patient presented

TABLE 1. Preoperative and postoperative JOA score, ADI, and CMA (mean \pm SD)

\begin{tabular}{lccc}
\hline \multicolumn{1}{c}{ Variable } & JOA & ADI $(\mathrm{mm})$ & $\mathrm{CMA}\left({ }^{\circ}\right)$ \\
\hline No. of patients & 44 & 41 & 41 \\
\hline Preop value & $13.03 \pm 1.45$ & $8.045 \pm 2.787$ & $124.130 \pm 12.655$ \\
\hline Postop value & $15.34 \pm 1.41$ & $0.780 \pm 1.267$ & $152.769 \pm 7.448$ \\
\hline t-test & 10.921 & 16.140 & 12.518 \\
\hline p value & 0.000 & 0.000 & 0.000 \\
\hline
\end{tabular}


with dyspnea 6 hours after surgery and died because the pedicle screw was inserted into the transverse foramen, thus leading to VA occlusion. ${ }^{3}$ Some researchers have reported that implanting a screw in the $\mathrm{C}-2$ pedicle isthmus likely squeezes the VA within the articular process. Such an implantation would lead to a tardive vertebrobasilar ischemic stroke, resulting in sudden breathing problems. ${ }^{2}$ In the present study, 2 patients with AAD presented with inspiratory dyspnea $0.5-1$ hour after recovering from anesthesia; however, no nerve injury was observed. Immediate tracheotomy was performed, after which the patients' breathing returned to normal. It is likely that the obstruction was in part of the upper respiratory tract. CT scanning performed 24 hours after surgery showed complete reduction in patients with AAD and satisfactory screw position without VA injury. MRI results 24 hours postoperatively showed no signal change of spinal ischemia, which remains to be further studied and explained. Careful attention should be paid to these findings in future clinical practice.

There are some limitations to the present study, such as its small sample size and retrospective nature. Also, we did not perform a comparison between the patients whose surgery was performed with the use of a 3D printed model and the patients for whom a 3D model was not available because there were not enough data. We would like to make a further comparison between these 2 surgical options in a future study if we can enroll a sufficient number of patients.

\section{Conclusions}

Posterior internal fixation performed with the assistance of an individually made 3D printed model is feasible and effective in treating CVJ abnormalities. Moreover, the use of these models offers advantages to surgeons, including the ability to perform a preoperative simulation for surgical planning and the use of a model for guidance during surgery, which can reduce intraoperative errors and increase intraoperative safety. The 3D printed model is clinically advantageous and may have great potential in guiding spinal surgery.

\section{Acknowledgments}

We give our gratitude to Prof. Feng-Zeng Jian, Department of Neurosurgery, Xuanwu Hospital, Capital Medical University, China.

This study was funded by the Collaborative Funds of Science and Technology Department of Guizhou Province and Guizhou Provincial People's Hospital (LH(2014)7007).

\section{References}

1. Goel A: Treatment of basilar invagination by atlantoaxial joint distraction and direct lateral mass fixation. J Neurosurg Spine 1:281-286, 2004

2. Gu SG, Du XR, Wang AH, Liu ZQ, Sun GZ, Cui ZC, et al: [Clinical application of internal fixation with lateral mass screw of atlas and axis pedicle screw.] Anat Clin 13:408411, 2008 (Chinese)

3. Jian FZ, Chen Z, Wrede KH, Samii M, Ling F: Direct posterior reduction and fixation for the treatment of basilar invagination with atlantoaxial dislocation. Neurosurgery 66:678-687, 2010

4. Kusaka M, Sugimoto M, Fukami N, Sasaki H, Takenaka M, Anraku T, et al: Initial experience with a tailor-made simulation and navigation program using a 3-D printer model of kidney transplantation surgery. Transplant Proc 47:596599, 2015

5. Menezes AH: Craniovertebral junction abnormalities with hindbrain herniation and syringomyelia: regression of syringomyelia after removal of ventral craniovertebral junction compression. J Neurosurg 116:301-309, 2012

6. Menezes AH: Craniovertebral junction database analysis: incidence, classification, presentation, and treatment algorithms. Childs Nerv Syst 24:1101-1108, 2008

7. Murphy SV, Atala A: 3D bioprinting of tissues and organs. Nat Biotechnol 32:773-785, 2014

8. Niikura T, Sugimoto M, Lee SY, Sakai Y, Nishida K, Kuroda $\mathrm{R}$, et al: Tactile surgical navigation system for complex acetabular fracture surgery. Orthopedics 37:237-242, 2014

9. Pande A: Craniovertebral junction abnormalities, in Thamburaj VA (ed): Textbook of Contemporary Neurosurgery. New Delhi: Jaypee, 2012, pp 443-456

10. Rengier F, Mehndiratta A, von Tengg-Kobligk H, Zechmann $\mathrm{CM}$, Unterhinninghofen R, Kauczor HU, et al: 3D printing based on imaging data: review of medical applications. Int J CARS 5:335-341, 2010

11. Rose AS, Webster CE, Harrysson OL, Formeister EJ, Rawal RB, Iseli CE: Pre-operative simulation of pediatric mastoid surgery with 3D-printed temporal bone models. Int J Pediatr Otorhinolaryngol 79:740-744, 2015

12. Silberstein JL, Maddox MM, Dorsey P, Feibus A, Thomas R, Lee BR: Physical models of renal malignancies using standard cross-sectional imaging and 3-dimensional printers: a pilot study. Urology 84:268-272, 2014

13. Smoker WR: Craniovertebral junction: normal anatomy, craniometry, and congenital anomalies. Radiographics 14:255-277, 1994

14. Wang C, Yan M, Zhou HT, Wang SL, Dang GT: Open reduction of irreducible atlantoaxial dislocation by transoral anterior atlantoaxial release and posterior internal fixation. Spine (Phila Pa 1976) 31:E306-E313, 2006

15. Yin Q, Ai F, Zhang K, Chang Y, Xia H, Wu Z, et al: Irreducible anterior atlantoaxial dislocation: one-stage treatment with a transoral atlantoaxial reduction plate fixation and fusion. Report of 5 cases and review of the literature. Spine (Phila Pa 1976) 30:E375-E381, 2005

\section{Disclosures}

The authors report no conflict of interest concerning the materials or methods used in this study or the findings specified in this paper.

\section{Author Contributions}

Conception and design: Gao. Acquisition of data: Wang, Xiong, Luo. Analysis and interpretation of data: Gao, Wang, Liu. Drafting the article: Liu, Luo. Critically revising the article: Gao. Reviewed submitted version of manuscript: Gao. Statistical analysis: Xiong. Study supervision: Gao.

\section{Correspondence}

Fangyou Gao, Department of Neurosurgery, Guizhou Provincial People's Hospital, No. 83, Zhongshan East Rd., Guiyang, Guizhou Province 550002, China. email: fy.gao@yahoo.com. 